Discrete Comput Geom 31:613-625 (2004)

DOI: $10.1007 / \mathrm{s} 00454-003-0819-2$

\title{
The Thirteen Spheres: A New Proof
}

\author{
Kurt M. Anstreicher \\ Department of Management Sciences, University of Iowa, \\ Iowa City, IA 52242, USA \\ kurt-anstreicher@uiowa.edu
}

\begin{abstract}
The "thirteen spheres problem," also known as the "Gregory-Newton problem," is to determine the maximum number of three-dimensional spheres that can simultaneously touch a given sphere, where all the spheres have the same radius. The history of the problem goes back to a disagreement between Isaac Newton and David Gregory in 1694. Using a combination of harmonic analysis and linear programming it can be shown that the maximum cannot exceed 13, but in fact 13 is impossible. The standard proof that the maximum is 12 uses an ad hoc construction that does not appear to extend to higher dimensions. In this paper we describe a new proof that uses linear programming bounds and properties of spherical Delaunay triangulations.
\end{abstract}

\section{Introduction}

For $n \geq 3$ let $S^{n-1}=\left\{x \in \mathfrak{R}^{n}: x^{T} x=1\right\}$, and $-1<z<1$. A finite set $\mathcal{C}=\left\{x_{i}\right\}_{i=1}^{M} \subset$ $S^{n-1}$ is called a spherical $z$-code if $x_{i}^{T} x_{j} \leq z$ for all $i \neq j$. For $z=\frac{1}{2},\left\{x_{i}\right\}$ correspond to contact points between $S^{n-1}$ and $M$ nonoverlapping spheres of radius 1 that are all incident to $S^{n-1}$. Maximizing the number $M$ of such spheres is called the kissing problem in dimension $n$, and the maximal $M$ is called the kissing number. The kissing problem in dimension 3 has a long history, going back to a celebrated disagreement between Isaac Newton and David Gregory in 1694. Newton believed that for $n=3$ the kissing number was 12 , while Gregory thought that 13 might be possible. In fact, $M=13$ is not possible, but the proof was quite long in coming. Several German papers in 1874/75 described approaches to the problem (see [2, first edition] and [19] for references), but the first proof accepted as being complete is due to Schütte and van der Waerden [17] in 1953. A subsequent proof by Leech [11] in 1956 is now the widely cited standard. Versions of Leech's proof also appear in the more recent references [2, first edition] and [19].

For general $n$ and $z$ there are several approaches that provide upper bounds on the size $M$ of a $z$-code $\mathcal{C} \subset S^{n-1}$; see, for example, [7] and [19]. In low dimensions the best known bounds are obtained using a combination of harmonic analysis and linear 
programming [7], [8], [10], [13], [19]. For $z=\frac{1}{2}$ this approach leads to a complete characterization of maximal codes in dimensions $n=8$ and 24 [4], [7], but for $n=3$ the result is a bound of 13. The proof that for $n=3$ the kissing number is 12 is not based on a general methodology, but instead uses an ad hoc construction to obtain a contradiction if $M=13$. The idea of the proof is simple and elegant, but there are many details that require verification. In fact the authors of [2, first edition] decided that the version published there was sufficiently incomplete that the entire chapter was removed in the second edition. A more serious drawback of the standard proof is that it seems impossible to extend it to higher dimensions, for two reasons. First, the proof is based on a construction that is tailored to $S^{2}$, and second, the final contradiction is obtained by showing that a certain graph is not planar. The kissing number is unknown in dimensions other than 3,8, and 24; for example, for $n=4$ a $\frac{1}{2}$-code with $M=24$ is known, but the linear programming bound is 25 .

In this paper we describe a new proof that the kissing number is 12 for $n=3$. Our approach is based on linear programming bounds and properties of the spherical Delaunay decomposition associated with $\mathcal{C}$. We do not claim that the proof here is "simpler" than the standard one [11]. However, because the new proof is based on structure associated with $\mathcal{C}$ that is not dependent on $n=3$, we believe that it has a much better chance of being extended to higher dimensions, for example to $n=4$.

An outline of the paper is as follows. In Section 2 we describe the linear programming bounds on $M$ that apply to a $z$-code $\mathcal{C} \subset S^{n-1}$. (We eventually utilize bounds based on $z>\frac{1}{2}$.) In Section 3 we consider the spherical Voronoi and Delaunay decompositions associated with $\mathcal{C}$. We describe an approach for obtaining lower bounds on the surface areas of spherical Delaunay triangles that gives another proof that $M \leq 13$. In Section 4 we use information from linear programming bounds to reduce the range of possible included angles that can occur in a spherical Delaunay triangle. This reduction provides a considerable improvement in the lower bound for the total surface area of a spherical Delaunay star (the spherical triangles containing a given point $x_{i} \in \mathcal{C}$ ) not consisting of five spherical triangles. The resulting lower bounds on the areas of Delaunay stars are sufficient to prove that $M=13$ is impossible.

Another recent proof for the 13 spheres problem is due to Böröczky [6] (see also Section 4.4 of [5]). Böröczky's proof also uses the spherical Delaunay triangulation, but in a different way from the proof presented here. In Böröczky's proof the spherical Delaunay triangulation is used to induce a network on $S^{2}$ that takes the place of the network used in Leech's proof. As in Leech's proof the network gives a decomposition of $S^{2}$ into spherical polygons, the total areas of which are shown to exceed the area of $S^{2}$. A similar proof is also given by Hsiang [9].

The kissing number problem is closely related to problems of packing spheres in $\Re^{n}$ [7], [19]. There has recently been considerable progress in settling the Kepler conjecture regarding the maximal density of sphere packings in dimension 3. See [12] for a detailed discussion and references.

\section{Linear Programming Bounds}

Let $\mathcal{C}=\left\{x_{i}\right\}_{i=1}^{M}$ be a spherical $z$-code in $\Re^{n}, n \geq 3$. In this section we describe a well-known linear programming bound for the size $M$ of such a code. The distance 
distribution of the code is the function $\lambda(\cdot):[-1,1] \rightarrow \Re_{+}$defined as

$$
\lambda(s)=\frac{\left|\left\{(i, j): x_{i}^{T} x_{j}=s\right\}\right|}{M} .
$$

It is then easy to see that

$$
\begin{aligned}
\lambda(1) & =1, \\
\sum_{-1 \leq s \leq z} \lambda(s) & =M-1, \\
\lambda(s) & \geq 0, \quad-1 \leq s \leq z .
\end{aligned}
$$

Let $\Phi_{k}(\cdot), k=0,1, \ldots$, denote the Gegenbauer, or ultraspherical, polynomials

$$
\Phi_{k}(t)=\frac{P_{k}^{(\beta, \beta)}(t)}{\left(\begin{array}{c}
k+\beta \\
k
\end{array}\right)},
$$

where $P_{k}^{(\beta, \beta)}$ is the Jacobi polynomial with $\beta=(n-3) / 2$ [1]. The normalization in (3) is chosen so that $\Phi_{k}(1)=1$ for all $k$. Using techniques from harmonic analysis it can be shown [10], [7, Chapter 9], [13], [19, Chapter 8] that

$$
1+\sum_{-1 \leq s \leq z} \lambda(s) \Phi_{k}(s) \geq 0, \quad k=1,2, \ldots
$$

From (2) and (4), using $k=1, \ldots, K$, a bound on $M$ can be obtained via the semi-infinite linear programming problem

$$
\begin{array}{ll}
\text { (LP) } \quad \max & \sum_{-1 \leq s \leq z} \lambda(s) \\
\text { s.t. } & \sum_{-1 \leq s \leq z} \lambda(s) \Phi_{k}(s) \geq-1, \quad k=1, \ldots, K, \\
& \lambda(s) \geq 0, \quad-1 \leq s \leq z .
\end{array}
$$

The dual of (LP) is the problem

$$
\begin{aligned}
\text { (LD) } \quad & \min \sum_{k=1}^{K} f_{k} \\
\text { s.t. } & \sum_{k=1}^{K} f_{k} \Phi_{k}(s) \leq-1, \quad-1 \leq s \leq z, \\
& f_{k} \geq 0, \quad k=1, \ldots, K .
\end{aligned}
$$

In practice it is impossible to solve (LD) exactly due to the infinite number of constraints. However a solution that is approximately feasible for (LD) can easily be adjusted to provide a valid bound on $M$, as described in the followng lemma.

Lemma 1 [7, p. 339]. Suppose that $0 \leq \varepsilon<1$, and for a given $n$ and $z, f \geq 0$ satisfies the constraints of $(\mathrm{LD})$ with the right-hand side of the constraints (5) relaxed to $-(1-\varepsilon)$. Then $M \leq 1+(1 /(1-\varepsilon))\left(\sum_{i=1}^{K} f_{k}\right)$ for any $z$-code in $\Re^{n}$. 
Proof. As described above $M \leq 1+v_{\mathrm{LD}}$, where $v_{\mathrm{LD}}$ denotes the optimal objective value in (LD). However, under the assumptions of the lemma $(1 /(1-\varepsilon)) f$ is feasible in (LD), and therefore $v_{\mathrm{LD}} \leq(1 /(1-\varepsilon))\left(\sum_{i=1}^{K} f_{k}\right)$.

In practice Lemma 1 can be used to obtain a valid bound on $M$ by discretizing $[-1, z]$ using $N+1$ points $s_{i}=-1+i \delta, i=0, \ldots, N$, where $\delta=(1+z) / N$. (LD) is then solved using the constraints corresponding to $\left\{s_{i}\right\}_{i=0}^{N}$, resulting in a solution $f \geq 0$. The value $\varepsilon$ required in the lemma is easily obtained by bounding the derivative of $\sum_{k=1}^{K} f_{k} \Phi_{k}(\cdot)$ on the intervals $\left[s_{i-1}, s_{i}\right], i=1, \ldots, N$. For $z=\frac{1}{2}$ this approach obtains the best known bounds on $M$ for dimensions $4 \leq n \leq 24$ [7], and leads to a complete characterization of the maximal $\frac{1}{2}$-codes in dimensions 8 and 24 [4], [7]. For $n=3$, using $K=16$ obtains a bound on $M$ of about 13.16. This bound can be reduced somewhat by imposing additional valid inequalities on the distance distribution $\lambda(\cdot)$, but to our knowledge a linear programming bound below 13 has never been obtained. (Better linear programming bounds on $M=|\mathcal{C}|$ can be obtained for special cases, for example the case that $\mathcal{C}$ is antipodal [3].)

\section{Spherical Delaunay Triangulations}

Let $\mathcal{C}=\left\{x_{i}\right\}_{i=1}^{M} \subset S^{2}, M \geq 4$. We assume throughout that the origin is in the interior of the convex hull of the points in $\mathcal{C}$; this is certainly the case if $\mathcal{C}$ is a maximal $\frac{1}{2}$-code. The spherical Voronoi cell associated with $x_{i}$ is the set

$$
V_{i}=\left\{x \in S^{2}:\left\|x, x_{i}\right\|_{g} \leq\left\|x, x_{j}\right\|_{g}, \forall j \neq i\right\},
$$

where $\|x, v\|_{g}=\cos ^{-1}\left(x^{T} v\right)$ denotes the geodesic distance on $S^{2}$. Each Voronoi cell is a spherical polygon whose sides are arcs of great circles, and $\bigcup_{i=1}^{M} V_{i}=S^{2}$.

The spherical Delaunay ${ }^{1}$ decomposition, which is dual to the decomposition of $S^{2}$ into Voronoi cells, is obtained by connecting each $x_{i}$ and $x_{j}$ such that $V_{i}$ and $V_{j}$ share a nontrivial boundary with a geodesic arc. If the points of $\mathcal{C}$ are in general position, then the Delaunay cells are all spherical triangles, and in any case spherical triangles can be obtained by adding additional arcs if necessary. We refer to a triangulation of $S^{2}$ so obtained as a spherical Delaunay triangulation (SDT). The following proposition describes the well-known Delaunay property that holds for an SDT.

Proposition 2 [5], [15]. Let $\left\{x_{i}, x_{j}, x_{k}\right\}$ be the vertices of a spherical triangle in an SDT. Then there is a spherical cap $\left\{x:\|x, v\|_{g} \leq R\right\}$ that circumscribes $\left\{x_{i}, x_{j}, x_{k}\right\}$ such that $R<\pi / 2$ and $\left\|x_{l}, v\right\|_{g} \geq R$ for all $x_{l} \in \mathcal{C}$.

The spherical Voronoi cells and Delaunay triangles for a typical $\mathcal{C}$ are depicted in Fig. 1. The spherical caps circumscribing the Delaunay triangles for the same $\mathcal{C}$ are depicted in Fig. 2. Figures 1 and 2 were produced by the ModeMap applet of Watson [18].

\footnotetext{
${ }^{1}$ The alternative translation Delone appears often in the literature.
} 


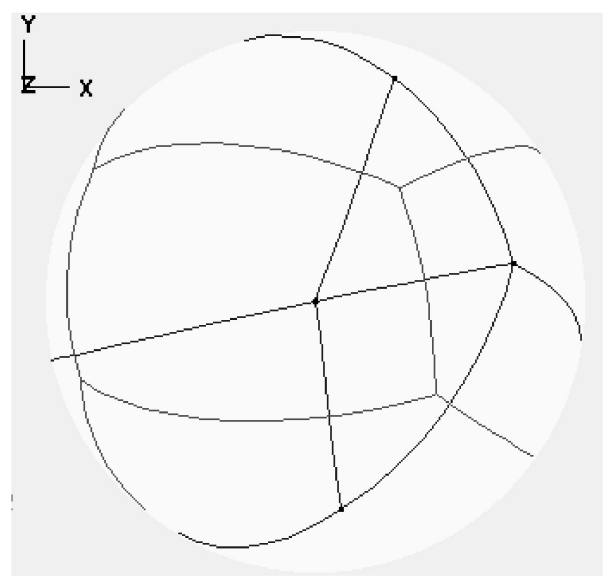

Fig. 1. Spherical Voronoi cells and Delaunay triangulation.

Consider a given spherical triangle in an SDT associated with $\mathcal{C}$. Let $a, b, c$ denote the geodesic lengths of the sides, and $\alpha, \beta, \gamma$ denote the corresponding opposite angles. We begin by collecting some useful formulas from spherical trigonometry [14], [16]. The law of cosines for spherical triangles is

$$
\cos c=\cos a \cos b+\sin a \sin b \cos \gamma .
$$

The area is given by the spherical excess

$$
E=\alpha+\beta+\gamma-\pi,
$$

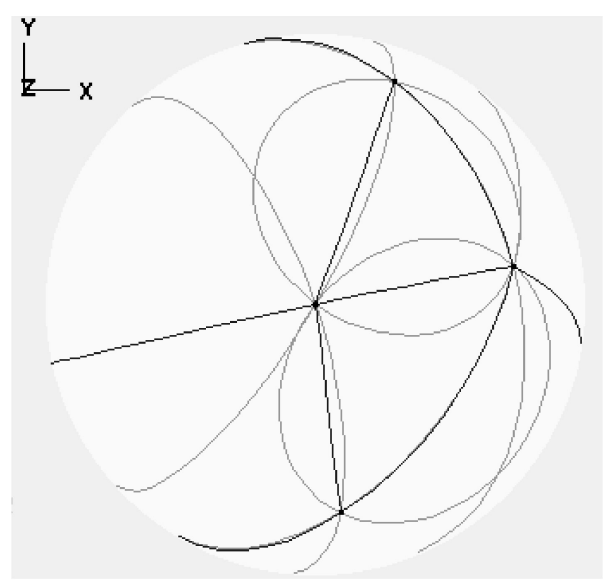

Fig. 2. Spherical Delaunay triangulation and circumscribing caps. 
where $\alpha+\beta$ can be obtained from $a, b$, and $\gamma$ using Gauss' formula

$$
\cos \frac{1}{2}(\alpha+\beta)=\frac{\sin \frac{1}{2} \gamma \cos \frac{1}{2}(a+b)}{\cos \frac{1}{2} c} .
$$

When $a=b$ it is straightforward to show that the spherical radius of a circumscribing spherical cap is given by

$$
\tan R=\frac{\tan \frac{1}{2} a}{\cos \frac{1}{2} \gamma}
$$

and in the general case $[14$, p. 78$]$

$$
\tan R=\frac{\tan \frac{1}{2} c}{\sin \left(\gamma-\frac{1}{2} E\right)}
$$

In what follows we consider $\gamma$ to be fixed, and choose $a$ and $b$ to minimize the area of the triangle, subject to valid constraints. We write $c(a, b \mid \gamma), E(a, b \mid \gamma)$, and $R(a, b \mid \gamma)$ to denote $c, E$, and $R$ as functions of $a$ and $b$ for a given $\gamma$. Since $\mathcal{C}$ is a $\frac{1}{2}$-code we certainly have $a \geq \pi / 3, b \geq \pi / 3, c(a, b \mid \gamma) \geq \pi / 3$. In addition we must have $R(a, b \mid \gamma) \leq \pi / 3$, since otherwise we could add another point at the center of the circumscribing cap and still have a $\frac{1}{2}$-code, which is impossible if $\mathcal{C}$ is maximal. To minimize the area of a spherical Delaunay triangle with included angle $\gamma$, associated with a maximal $\frac{1}{2}$-code $\mathcal{C}$, we are thus led to the problem

$$
\begin{array}{ll}
\left(P_{\gamma}\right) \quad \min & E(a, b \mid \gamma) \\
\text { s.t. } \quad & c(a, b \mid \gamma) \geq \frac{\pi}{3}, \\
& R(a, b \mid \gamma) \leq \frac{\pi}{3}, \\
& a \geq b \geq \frac{\pi}{3} .
\end{array}
$$

In the next two lemmas we give a complete characterization of the solution of $\left(P_{\gamma}\right)$. Let $\gamma^{0}=\cos ^{-1}\left(\frac{1}{3}\right)$.

Lemma 3. $\left(P_{\gamma}\right)$ is infeasible for $\gamma>2 \gamma^{0}$. For $\gamma^{0} \leq \gamma \leq 2 \gamma^{0}$ the solution of $\left(P_{\gamma}\right)$ is $a=b=\pi / 3$.

Proof. It is obvious that the area $E(a, b \mid \gamma)$ is monotonically increasing in $a$ and $b$, and therefore the minimum possible value corresponds to $a=b=\pi / 3$. Using (6) and (9) it is straightforward to compute that $c(\pi / 3, \pi / 3 \mid \gamma) \geq \pi / 3$ for $\gamma \geq \gamma^{0}$, and $R(\pi / 3, \pi / 3 \mid \gamma) \leq \pi / 3$ for $\gamma \leq 2 \gamma^{0}$. Moreover for $\gamma \geq \pi / 2$ it is easy to see that $R(a, b \mid \gamma)$ is monotonically increasing in $a$ and $b$, and therefore $\left(P_{\gamma}\right)$ is infeasible for $\gamma>2 \gamma^{0}$.

Lemma 4. ( $\left.P_{\gamma}\right)$ is infeasible for $\gamma<\frac{1}{2} \gamma^{0}$. For $\frac{1}{2} \gamma^{0} \leq \gamma \leq \gamma^{0}$ the solution of $\left(P_{\gamma}\right)$ has $b=c=\pi / 3$. 
Proof. Consider $\left(P_{\gamma}\right)$ with the constraint on $R$ ignored. We will show that the solution of this problem has $b=c(a, b \mid \gamma)=\pi / 3$, and then consider the effect of the constraint on $R$. To start we argue that $b=\pi / 3$. It is straightforward to compute that

$$
\begin{aligned}
& \frac{\partial \cos c(a, b \mid \gamma)}{\partial a}+\frac{\partial \cos c(a, b \mid \gamma)}{\partial b}=(\cos \gamma-1) \sin (a+b), \\
& \frac{\partial \cos c(a, b \mid \gamma)}{\partial a}-\frac{\partial \cos c(a, b \mid \gamma)}{\partial b}=-(\cos \gamma+1) \sin (a-b) .
\end{aligned}
$$

Suppose that we have $a \geq b>\pi / 3$ with $c(a, b \mid \gamma) \geq \pi / 3$. From (11a), if $a+b \geq \pi$, then simultaneously decreasing $a$ and $b$ will increase $c(a, b \mid \gamma)$ while also decreasing $E(a, b \mid \gamma)$. This process could be continued until either $b=\pi / 3$ or $a+b=\pi$. In the first case we are done, so consider now the case where $a \geq b>\pi / 3, a+b \leq \pi$. From (11b) simultaneously increasing $a$ and decreasing $b$ will increase $c(a, b \mid \gamma)$. Moreover, from (8) this same operation will decrease $\alpha+\beta$, and therefore also $E(a, b \mid \gamma)$, until we reach $b=\pi / 3$.

We have now shown that the solution of $\left(P_{\gamma}\right)$, with the constraint on $R$ ignored, has $b=\pi / 3$. However, decreasing $a$ will decrease $E(a, b \mid \gamma)$, and $c(\pi / 3, \pi / 3 \mid \gamma)<\pi / 3$ for $\gamma<\gamma^{0}$. We must therefore have $c(a, b \mid \gamma)=\pi / 3$ in the solution, for $\gamma \leq \gamma^{0}$. This completes the analysis of the problem with the constraint on $R$ ignored.

Next we consider the effect of the constraint on $R$. For $b=c=\pi / 3$, (6) implies that

$$
\cos a=\frac{1-3 \cos ^{2} \gamma}{1+3 \cos ^{2} \gamma}
$$

The analog of (9) gives

$$
\tan R=\frac{1}{\sqrt{3} \cos \frac{1}{2} \alpha}
$$

so $R \leq \pi / 3$ is equivalent to $\cos \frac{1}{2} \alpha \geq \frac{1}{3}$, or $\cos \alpha \geq-7 / 9$. From the analog of (6) we obtain

$$
\cos a=\frac{1}{4}+\frac{3}{4} \cos \alpha
$$

so $\cos \alpha \geq-7 / 9$ is equivalent to

$$
\cos a \geq-1 / 3
$$

Comparing (12) and (14) we conclude that the solution with $b=c=\pi / 3$ results in $R \leq \pi / 3$ for $\gamma \geq \cos ^{-1} \sqrt{\frac{2}{3}}=\frac{1}{2} \gamma^{0}$. However, for all $\gamma \leq \gamma^{0}$ the solution with $b=c=\pi / 3$ simultaneously minimizes the numerator of the right-hand side in (10) while maximizing the denominator, and therefore $\left(P_{\gamma}\right)$ is infeasible for $\gamma<\frac{1}{2} \gamma^{0}$.

For $\frac{1}{2} \gamma^{0} \leq \gamma \leq 2 \gamma^{0}$ we can easily obtain the minimal area associated with the solution of $\left(P_{\gamma}\right)$ by computing $\alpha$ and $\beta$. In the case of $\gamma^{0} \leq \gamma \leq 2 \gamma^{0}$ we have $a=b=$ $\pi / 3$, from Lemma 3. Therefore $\alpha=\beta$, and $\cos c=\frac{1}{4}+\frac{3}{4} \cos \gamma$, from (6). Using (8) and ordinary trigonometric identities we obtain

$$
\cos \alpha=\cos \beta=\sqrt{\frac{1-\cos \gamma}{5+3 \cos \gamma}} .
$$




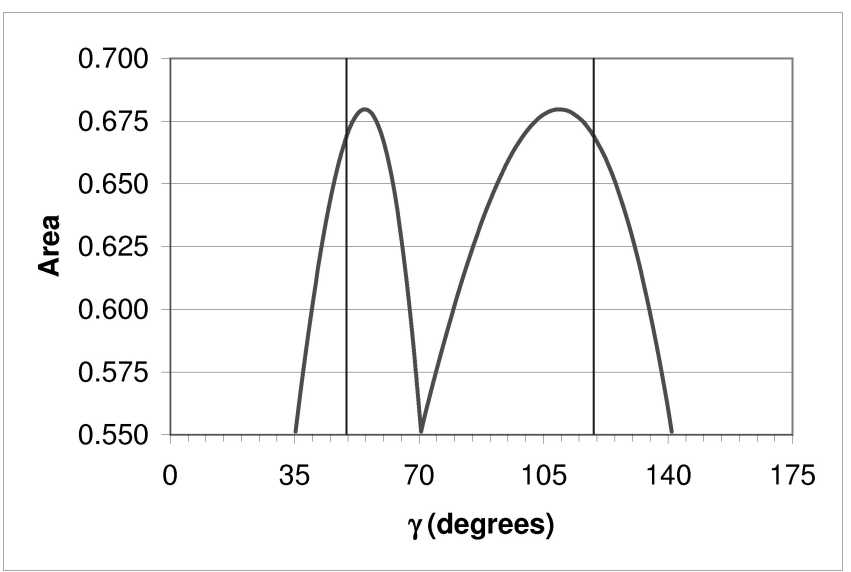

Fig. 3. Minimal area of a Delaunay triangle with included angle $\gamma$.

For $\frac{1}{2} \gamma^{0} \leq \gamma \leq \gamma^{0}$ we have $b=c=\pi / 3$, from Lemma 4. Therefore $\beta=\gamma$, and (12) and (13) together imply that

$$
\cos \alpha=\frac{1-5 \cos ^{2} \gamma}{1+3 \cos ^{2} \gamma} .
$$

From (15) and (16) we can easily compute the solution value of $\left(P_{\gamma}\right)$ as a function of $\gamma$, using (7). In Fig. 3 we plot the resulting minimal area. (The vertical lines in the figure are explained in the next section and can be ignored for the moment.) The minimal value of $3 \gamma^{0}-\pi \approx 0.55129$ occurs at $\gamma^{0}, \frac{1}{2} \gamma^{0}$, and $2 \gamma^{0}$. Since the total surface area of $S^{2}$ is $4 \pi \approx 12.5664$, this lower bound implies that there can be at most 22 spherical Delaunay triangles in an SDT associated with $\mathcal{C}$. Let $E$ and $F$ denote the number of edges and faces (triangles) in this SDT. From Euler's theorem we have $F+M=E+2$, and $3 F=2 E$ for a triangulation. Then $M=\frac{1}{2} F+2$, so $F \leq 22$ gives another proof that $M \leq 13$.

Note that for $\gamma=\gamma^{0}$ the spherical triangle of minimal area is equilateral, with edges of geodesic length $\pi / 3$. In [5] it is shown directly that the area of this triangle $(\approx 0.55129)$ is minimal among all possible spherical triangles in an SDT induced by a maximal $\frac{1}{2}$-code.

Let $n_{i}$ denote the number of edges incident to $x_{i}$ in the SDT (equal to the number of Voronoi cells sharing a nontrivial border with $V_{i}$ if the points of $\mathcal{C}$ are in general position). If $M=13$, then

$$
\sum_{i=1}^{13} n_{i}=2 E=66
$$

implying that $n_{i} \neq 5$ for at least one $i$. For each $x_{i}$ the spherical Delaunay star at $x_{i}$ is the union of the spherical Delaunay triangles in the SDT that have $x_{i}$ as a vertex. Our proof is based on examining the total surface area of spherical Delaunay stars, in particular the star at an $x_{i}$ with $n_{i} \neq 5$. Unfortunately the analysis of $\left(P_{\gamma}\right)$ above is not sufficient to produce a contradiction based on the total surface area of such a star. For 
each spherical triangle in the star at a point $x_{i}$ we take $\gamma$ to be the angle between the two edges incident to $x_{i}$. For $n_{i}=5$ we can then take four spherical triangles with $\gamma=\gamma^{0}$, and one with $\gamma=2 \pi-4 \gamma^{0}$, to get a lower bound of the total surface area of the star equal to about 2.7965. For $n_{i}=6$ we can use two triangles with $\gamma=\frac{1}{2} \gamma^{0}$ in place of one of the triangles with $\gamma=\gamma^{0}$ to get a lower bound of about 3.3478. Summing the bounds on the areas of the stars for 12 points $x_{i}$ with $n_{i}=5$, and one with $n_{i}=6$, and dividing by 3 then obtains a lower bound on the total area of the triangles in the SDT of about 12.3019 . This is greater than $22(0.55129) \approx 12.1284$ but still less than $4 \pi$. In fact the same lower bound can be achieved with any combination of $n_{i}$ having $3 \leq n_{i} \leq 9$, $i=1, \ldots, 13$, satisfying (17).

In the next section we show how the analysis of an SDT can be improved by incorporating information from the (LP) bounds described in Section 2.

\section{The Kissing Number for $n=3$}

As described in the previous two sections, bounds based on linear programming and an analysis of SDTs both individually suffice to prove that $M=|\mathcal{C}| \leq 13$ for a $\frac{1}{2}$-code $\mathcal{C}$ in $S^{2}$. In this section we show how these two methodologies can be combined to give a new proof that $M \leq 12$. Our approach is based on strengthening the constraint on $R$ used in the formulation of problem $\left(P_{\gamma}\right)$ of the previous section. Recall that the logic of this constraint is based on the fact that if $\mathcal{C}$ is a maximal $\frac{1}{2}$-code, then it is impossible to have $R \geq \pi / 3$ for a spherical Delaunay triangle since otherwise $\mathcal{C}$ could be augmented to obtain a larger $\frac{1}{2}$-code. We now consider the possibility of adding a point to $\mathcal{C}$ such that the augmented code $\mathcal{C}^{+}$is a $z$-code, with $z>\frac{1}{2}$. The following lemma is useful for obtaining valid constraints on the distance distribution of the augmented code $\mathcal{C}^{+}$.

Lemma 5. Suppose that a spherical triangle has $c \geq \pi / 3,0 \leq z_{a} \leq \cos a \leq \cos b \leq$ $z_{b}<1$. Then

$$
\cos \gamma \leq \max \left\{\frac{\frac{1}{2}-z_{a}^{2}}{1-z_{a}^{2}}, \frac{\frac{1}{2}-z_{a} z_{b}}{\sqrt{\left(1-z_{a}^{2}\right)\left(1-z_{b}^{2}\right)}}\right\} .
$$

Moreover if $z_{a}<\cos a$, then the above inequality is also strict.

Proof. Consider the problem to minimize $\gamma$ subject to the given constraints on $a, b$, and $c$. By assumption $b \leq a<\pi / 2$, so (11a) implies that for any $\gamma, a$ and $b$ can be simultaneously increased resulting in an increase in $c$. Thus we may assume that $\cos a=z_{a}$. Using (6) and ordinary trigonometric identities we conclude that

$$
\cos ^{2} \gamma \leq \frac{\left(\frac{1}{2}-z_{a} \cos b\right)^{2}}{\left(1-z_{a}^{2}\right)\left(1-\cos ^{2} b\right)}
$$

The numerator of the right-hand side of (18) is convex in $\cos b$, while the denominator is positive and concave in $\cos b$. Therefore the ratio is a quasiconvex function of $\cos b$, implying that the maximum must occur at one of the bounds $z_{b}$ or $z_{a}$. This proves the 
weak inequality of the lemma. The strict inequality is an immediate consequence of (11a); if $\cos a>z_{a}$, then $a$ and $b$ can both be increased, resulting in a strict increase in $c$, allowing a strict decrease in $\gamma$.

Note that for $z_{a}=\frac{1}{7}, z_{b}=\frac{1}{2}$ Lemma 5 implies that if $\pi / 3 \leq b \leq a<\cos ^{-1}\left(\frac{1}{7}\right)$ and $c \geq \pi / 3$, then $\gamma>\pi / 6$. This fact is an important element of Leech's proof [11].

Lemma 6. Suppose that $\mathcal{C}^{+}=\left\{x_{i}\right\}_{i=1}^{14}$ is a $z$-code, $0.5<z \leq 0.66$, where $\mathcal{C}=\left\{x_{i}\right\}_{i=1}^{13}$ is a $\frac{1}{2}$-code. Let

$$
z_{5}=\frac{\sqrt{\sin ^{2}(\pi / 5)-\frac{1}{4}}}{\sin (\pi / 5)} \approx 0.5257 .
$$

Then the distance distribution for $\mathcal{C}^{+}$satisfies the constraints:

1. $\sum_{0.2<s \leq z} \lambda(s) \leq 5$,

2. $\sum_{0.5<s \leq z} \lambda(s) \leq \frac{5}{7}$,

3. $\sum_{z 5<s \leq z} \lambda(s) \leq \frac{4}{7}$, if $z>z_{5}$.

Proof. For part 1 we apply Lemma 5 with $z_{a}=0.2, z_{b}=0.66$, and obtain $\gamma>\pi / 3$. It follows that for each $x_{i}, i=1, \ldots, 14$, there can be at most five $x_{j}$ having $0.2<$ $x_{i}^{T} x_{j} \leq 0.66$. Part 2 follows from this same observation, and the fact that $\mathcal{C}$ is a $\frac{1}{2}$-code. For part 3 we use $z_{a}=z_{5}, z_{b}=0.66$ and obtain $\gamma>2 \pi / 5$, so there can be at most four $x_{i}, i=1, \ldots, 13$, with $z_{5}<x_{i}^{T} x_{14} \leq 0.66$.

Theorem 7. Suppose that $\mathcal{C}=\left\{x_{i}\right\}_{i=1}^{13}$ is a $\frac{1}{2}$-code. Then $\cos R \geq 0.6595$ for any Delaunay triangle in an SDT associated with $\mathcal{C}$.

Proof. We consider problem (LP) from Section 2 with $0.5<z \leq 0.66, K=10$, and the additional constraints on $\lambda(\cdot)$ from Lemma 6 . Forming the dual problem, discretizing the constraints, and adjusting the solution value as described in Lemma 1, we obtain a bound $^{2}$ strictly less than 14 for $0.5<z \leq 0.6595$. It follows from the Delaunay property that $R \leq \cos ^{-1}(0.6595)$ for any Delaunay triangle associated with $\mathcal{C}$, since otherwise we could construct an augmented code $\mathcal{C}^{+},\left|\mathcal{C}^{+}\right|=14$ whose distance distribution was feasible for all the constraints of (LP).

Let $\left(P_{\gamma, z}\right)$ denote problem $\left(P_{\gamma}\right)$, from the previous section, but with the constraint on $R$ replaced by

$$
\cos R(a, b \mid \gamma) \geq z
$$

Lemmas 3 and 4 can easily be generalized to the case of $z>\frac{1}{2}$, as described below.

\footnotetext{
${ }^{2}$ Full details of the solutions of the linear programming problems are available from the author. A bound strictly less than 14 is actually obtained for $z$ up to approximately 0.65976 .
} 
Lemma 8. Suppose that $\frac{1}{2} \leq z \leq \sqrt{\frac{2}{3}}$. Let $\gamma^{+}=2 \cos ^{-1} \sqrt{z^{2} / 3\left(1-z^{2}\right)}$. Then $\left(P_{\gamma, z}\right)$ is infeasible for $\gamma>\gamma^{+}$. For $\gamma^{0} \leq \gamma \leq \gamma^{+}$the solution of $\left(P_{\gamma, z}\right)$ is $a=b=\pi / 3$.

Proof. The proof follows that of Lemma 3, except that $\cos R(\pi / 3, \pi / 3 \mid \gamma) \geq z$ for $\gamma \leq \gamma^{+}$. For $\gamma<\pi / 2$ it is not obvious that $R(a, b \mid \gamma)$ is monotone in $a$ and $b$. However, it is easy to show that $a=b=\pi / 3$ minimizes $c(a, b \mid \gamma)$ while also minimizing $E(a, b \mid \gamma)$. For $\gamma \leq \pi / 2$ this implies that $a=b=\pi / 3$ simultaneously minimizes the numerator of the right-hand side of (10) while maximizing the denominator, again implying that $\left(P_{\gamma, z}\right)$ is infeasible for $\gamma>\gamma^{+}$.

Lemma 9. Suppose that $\frac{1}{2} \leq z \leq \sqrt{\frac{2}{3}}$. Let $\gamma^{-}=\cos ^{-1} \sqrt{1-\frac{4}{3} z^{2}}$. Then $\left(P_{\gamma, z}\right)$ is infeasible for $\gamma<\gamma^{-}$. For $\gamma^{-} \leq \gamma \leq \gamma^{0}$ the solution of $\left(P_{\gamma, z}\right)$ has $b=c=\pi / 3$.

Proof. The proof is identical to that of Lemma 4, except that $b=c=\pi / 3$ results in $\cos R \geq z$ for $\gamma \geq \gamma^{-}$.

In Fig. 3 the two vertical lines correspond to the values $\gamma^{+}$and $\gamma^{-}$from Lemmas 8 and 9 , respectively, using $z=0.6595$. It is obvious from the figure that use of $z=0.6595$, as opposed to the original $z=0.5$, considerably reduces the range of possible values of the included angle $\gamma$. This reduction has a substantial effect on the lower bound for the area of a spherical Delaunay star at a point $x_{i}$ having $n_{i} \neq 5$ triangles. From the figure it is also apparent that the area bounds for $\gamma=\gamma^{+}$and $\gamma=\gamma^{-}$coincide. In fact it is easy to show that this is the case for any $\frac{1}{2} \leq z \leq \sqrt{\frac{2}{3}}$.

Consider now an SDT associated with a $\frac{1}{2}$-code $\mathcal{C}=\left\{x_{i}\right\}_{i=1}^{13}$. Using Lemma 8 and (15), the minimal area of a spherical Delaunay triangle in this SDT, with included angle $\gamma^{0} \leq \gamma \leq \gamma^{+}$, is bounded from below by

$$
f^{+}(\gamma)=\gamma+2 \cos ^{-1} \sqrt{\frac{1-\cos \gamma}{5+3 \cos \gamma}}-\pi .
$$

From Lemma 9 and (16), the minimal area of a spherical Delaunay triangle in the SDT, with included angle $\gamma^{-} \leq \gamma \leq \gamma^{0}$, is bounded from below by

$$
f^{-}(\gamma)=2 \gamma+\cos ^{-1} \frac{1-5 \cos ^{2} \gamma}{1+3 \cos ^{2} \gamma}-\pi
$$

Since $f^{+}(\cdot)$ and $f^{-}(\cdot)$ are both concave on their domains of definition, the minimum possible area for a spherical Delaunay star at $x_{i}$ containing $n_{i}$ triangles corresponds to all but one of the triangles in the star having included angle $\gamma$ equal to either $\gamma^{0}, \gamma^{+}$, or $\gamma^{-}$, with the last chosen to make the sum of the angles equal to $2 \pi$. Enumerating the very small number of possibilities, continuing to use $z=0.6595$, we obtain the bounds in Table 1. (Note that $4 \leq n_{i} \leq 7$ follows from $\gamma^{+}<2 \pi / 3, \gamma^{-}>\pi / 4$.) In the table we also report the number of triangles with included angles equal to $\gamma^{0}, \gamma^{+}$, and $\gamma^{-}$ that achieve the minimum total area, and the minimal average area (total area divided by 
Table 1. Bounds for areas of Delaunay stars.

\begin{tabular}{cccccc}
\hline Triangles & $\gamma^{0}$ & $\gamma^{+}$ & $\gamma^{-}$ & Area & Average \\
\hline 4 & 2 & 1 & 0 & 2.44178 & 0.61044 \\
5 & 4 & 0 & 0 & 2.79648 & 0.55929 \\
6 & 2 & 0 & 3 & 3.66747 & 0.61124 \\
7 & 0 & 0 & 6 & 4.66593 & 0.66656 \\
\hline
\end{tabular}

number of triangles). The area and average area are rounded down to give valid lower bounds.

Theorem 10. Let $\mathcal{C}=\left\{x_{i}\right\}_{i=1}^{M}$ be a $\frac{1}{2}$-code in $S^{2}$. Then $M \leq 12$.

Proof. Assume that $M=13$. Recall that the minimum possible area for a spherical Delaunay triangle in an SDT associated with $\mathcal{C}$ is $3 \gamma^{0}-\pi>0.55128$. If there is a $j$ with $n_{j}=7$, then using the bound from Table 1 the total area of the triangles in the SDT would be at least $4.66593+15(0.55128)=12.93513>4 \pi$. Therefore there can be no $j$ with $n_{j}=7$. Next suppose that there is a $j$ with $n_{j}=4$. Then there must be at least two points $x_{i}$ with $n_{i}=6$. Summing over the Delaunay stars, the total area is bounded from below by

$$
\frac{2.44178+2(3.66747)+50(0.55929)}{3} \approx 12.58>4 \pi
$$

so there can be no $j$ with $n_{j}=4$. It must then be the case that $n_{j}=6$ for one $j$, and $n_{i}=5$ for all $i \neq j$. For convenience suppose that $j=1$, and $\left\{x_{i}\right\}_{i=2}^{7}$ are the six other nodes in the star at $x_{1}$. The area of the star at $x_{1}$ is bounded from below by 3.66747. For $i=2, \ldots, 7$ the Delaunay star at $x_{i}$ contains two spherical triangles in common with the star at $x_{1}$, and three other triangles. Summing the areas of these six stars we get twice the area of the star at $x_{1}$, plus the areas of 18 spherical triangles. Finally, for $i=8, \ldots, 13$ the area of the star at $x_{i}$ is bounded from below by 2.79648. Summing over all the stars, the total area is bounded from below by

$$
\frac{3(3.66747)+18(0.55128)+6(2.79648)}{3} \approx 12.56811>4 \pi,
$$

and therefore $M=13$ is impossible.

Although the area bounds in Table 1 are sufficient to complete the proof of Theorem 10, it is worth noting that the bound for the area of a star with $n_{i}=6$ from Table 1 clearly cannot be attained. This bound corresponds to three spherical triangles with $\gamma=\gamma^{-}$, $b=\pi / 3, a \approx 1.6862$, two with $\gamma=\gamma^{0}, a=b=\pi / 3$, and one with $\gamma$ slightly less that $\gamma^{0}, b=\pi / 3$, and $a$ slightly greater than $\pi / 3$. It is obvious that it is impossible to assemble six such spherical triangles into a spherical star. It is also interesting to note that the last case considered in the proof, $n_{j}=6$ for one $j$, and $n_{i}=5$ for all $i \neq j$, could alternatively be excluded using a graph-theoretic argument. The dual of this SDT would be a cubic, planar graph that divides $S^{2}$ into one hexagon and 12 pentagons. It is 
easy to show that no such graph can exist. Leech's proof also uses arguments based on area to eliminate all but one case, which corresponds to an unrealizable planar graph. However, the area arguments here are completely different, as is the graph in the final case.

\section{References}

1. M. Abramowitz and I.A. Stegun. Handbook of Mathematical Functions. National Bureau of Standards Applied Mathematics Series 55. U.S. Department of Commerce, Washington, DC, 1972.

2. M. Aigner and G. Ziegler. Proofs from THE BOOK. Springer-Verlag, Berlin, 1998; second edition, Springer-Verlag, Berlin, 2001.

3. K.M. Anstreicher. Improved linear programming bounds for antipodal spherical codes. Discrete Comput. Geom. 28 (2002), 107-114.

4. E. Bannai and N.J.A. Sloane. Uniqueness of certain spherical codes. Canad. J. Math. 33 (1981), 437-449.

5. K. Böröczky Jr. Finite Packing and Covering. A. Rényi Institute of Mathematics, Hungarian Academy of Sciences, Budapest, 2002.

6. K. Böröczky. The Newton-Gregory problem revisited. In Discrete Geometry: In Honor of W. Kuperberg's 60th Birthday, A. Bezdek ed. Marcel Dekker, New York, to appear.

7. J.H. Conway and N.J.A. Sloane. Sphere Packings, Lattices and Groups, third edition. Springer-Verlag, New York, 1999.

8. P. Delsarte, J.-M. Goethals, and J.J. Seidel. Spherical codes and designs. Geom. Dedicata 6 (1977), 363-388.

9. W.-Y. Hsiang. Least Action Principle of Crystal Formation of Dense Packing Type and Kepler's Conjecture. World Scientific, Singapore, 2001.

10. G.A. Kabatiansky and V.I. Levenshtein. Bounds for packings on a sphere and in space. Problems Inform. Transmission 14 (1978), 1-17.

11. J. Leech. The problem of the thirteen spheres. Math. Gaz. 40 (1956), 22-23.

12. J.C. Lagarias. Bounds for local density of sphere packings and the Kepler conjecture. Discrete Comput. Geom. 27 (2002), 165-193.

13. V.I. Levenshtein. Universal bounds for codes and designs. In Handbook of Coding Theory, Volume 1, V.S. Pless and W.C. Huffman, eds. North-Holland, Amsterdam, 1998, pp. 499-648.

14. E. Miller. Plane and Spherical Trigonometry, third edition. Leach, Shewell and Sanborn, Boston, MA, 1894.

15. H.-S. Na, C.-N. Lee, and O. Cheong. Voronoi diagrams on the sphere. Department of Mathematics, Pohang University of Science and Technology, Korea, 2001.

16. H.L. Pearson. Formulas from algebra, trigonometry, and analytic geometry. In Handbook of Applied Mathematics, second edition, C.E. Pearson, ed. Van Nostrand Reinhold, New York, 1983, Chapter 1.

17. K. Schütte and B.L. van der Waerdan. Das Problem der dreizehn Kugeln. Math. Ann. 53 (1953), 325-334.

18. D.F. Watson. ModeMap: An implementation of natural neighbor interpolation on the sphere. P.O. Box 734, Claremont, WA 6010, Australia, 1998.

19. C. Zong. Sphere Packings. Springer-Verlag, Berlin, 1999.

Received November 25, 2002, and in revised form June 6, 2003. Online publication February 9, 2004. 